\title{
17/7 Translocation trisomy: practical vindication of a model of sickness
}

\author{
J F HIGGS, E RIVLIN, AND D C TAYLOR
}

Department of Child and Adolescent Psychiatry, Booth Hall Children's Hospital; Central Manchester Health Authority, St Mary's Hospital, and University of Manchester, Manchester

SUMmaRY A girl was seen by a psychiatrist for relentless rubbing of her eyes and difficult behaviour. A proper diagnosis of her sickness required an understanding of the cause of her physical stigmata, which disclosed a previously undescribed chromosome anomaly (partial trisomy 17p); her mental handicap and behavioural disorder, which were partly a function of parental despair; and the predicament of her father and other carrier relatives who were identified through this child. Explanation or alteration of each component of sickness was necessary to achieve beneficial change.

So called medical models of the management of patients are under pressure in child psychiatry and mental retardation clinics. The pressure comes partly from growing aspirations of professional colleagues in other disciplines and partly from a failure of psychiatrists to justify themselves as physicians. Taylor provided a model of sickness that allows for a holistic approach to psychiatric patients without neglecting the pre-eminently medical component of care of the sick, that of correct diagnosis. ${ }^{12}$ Diagnosis is not, however, confined to structural disease (things) but extends to illness (the way that the problem is experienced) and the predicament in which it is experienced and to which it gives rise.

A small girl, aged 5, was sent by an ophthalmologist to a psychiatrist because she persisted in rubbing her right eye with such severity that she risked corneal abrasion, infection, and amaurosis.

\section{Presentation}

The psychiatrist had not been told of the girl's startlingly abnormal appearance, her obvious mental retardation, or her grossly restricted and abnormal range of behaviour. This was potentially unnerving to the psychiatrist. The parents' understanding of the nuances of the language as an instrument of communication was impaired. They described how they were taking her to 12 different specialist clinics and also enjoyed many professional visitors to the home. The clinics offered a variety of advice that was often mutually incompatible. Local agencies had agreed among themselves that the mother had not come to terms with her child's handicap or need for special care.

Her parents were apparently healthy and nonconsanguineous. Her mother was aged 25 at the time of the child's birth and had had one previous pregnancy, leading to the birth of an apparently normal boy, now aged 8. During the mother's pregnancy with the daughter retardation of intrauterine growth was noted at 17 weeks (12 weeks' size) and confirmed at 36 weeks by ultrasound scan (32 weeks' size). At 37 weeks a lower segment caesarian operation was performed because of the poor growth and oligohydramnios. Birthweight was $2120 \mathrm{~g}$, and her condition at birth was good, but she was obviously dysmorphic and was extensively investigated. Appropriate follow up care was given for a retarded child but no diagnosis was made.

On examination at the age of 5 the girl was an unattractive, dysmorphic child with an unappealing behavioural repertoire. She had an unsteady gait, microcephaly, and microphthalmia among other minor abnormalities (Figs. 1 and 2 and Appendix). In her mother's presence she displayed angry and anxious attachment with tantrums of temper and severe rubbing of her eyes if her wishes were not gratified. She did not smile and was irritable.

Genetic and behavioural components to the predicament were present. These were initially denied by the parents, but by persistent domiciliary psychiatric nursing and brief inpatient treatment of behaviour the true nature of the family emerged for the first time. The father had relatives with similar 


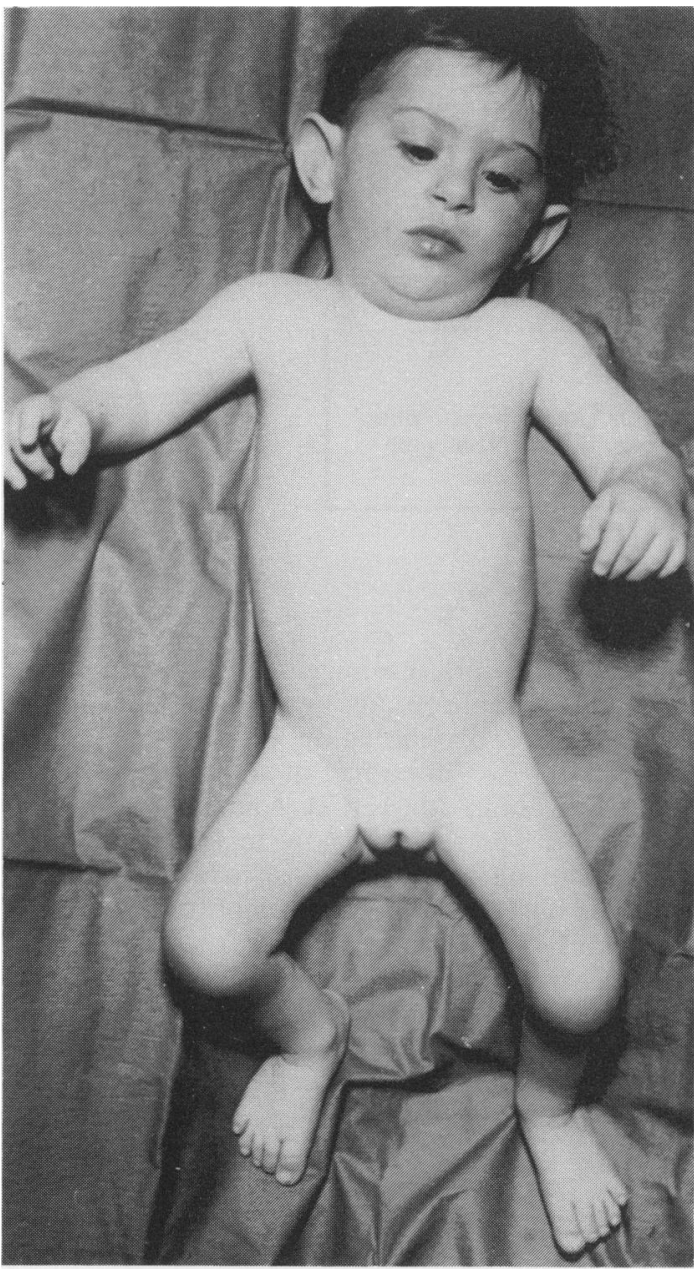

Fig. 1 Propositus at age 11 months.

problems. Perhaps dimly aware of his genetic responsibility he took refuge in drinking binges and violence towards his wife: the marriage was breaking down. The child's maternal grandmother, more dependent than supportive, collapsed under stress into 'attacking'. As a result the child's mother had exhausted her parenting capacities and resources in dealing with her handicapped child.

\section{Investigation}

Among other investigations a referral back to the department of genetics was made itemising the reasons for postulating a possible chromosomal anomaly. Further analysis showed a partial trisomy $17 p$ with a tiny monosomy $7 q$. The father was found

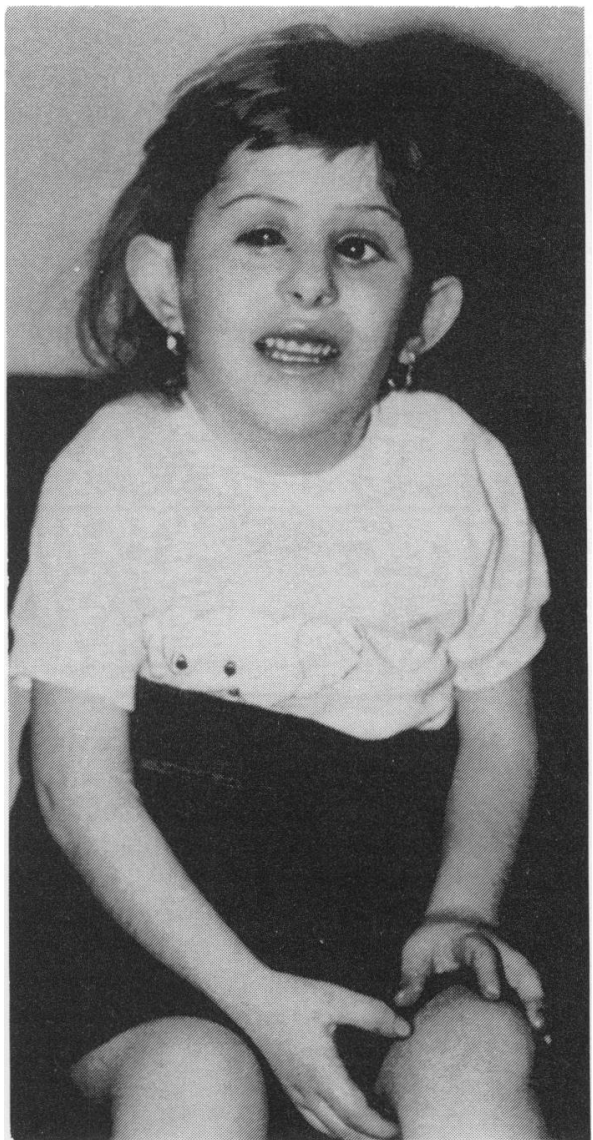

Fig. 2 Propositus at age 5 years.

to have a balanced translocation such that part of the short arm of chromosome 17 had become translocated to the long arm of chromosome 7 and presumably the tiny terminal portion of the long arm of chromosome 7 had transferred on to the stump of the short arm of 17. The girl had received the normal chromosome 17 and the chromosome 7 with the translocated part of $17 \mathrm{p}$ from her father.

The girl's brother also had a balanced translocation (Fig. 3) as did the mother of the girl's cousin, whose karyotype abnormality had been recognised at another hospital in another part of the country. This karyotype abnormality was identical to the girl's. The girl's cousin was seen at age 9. His phenotype was almost identical to that of the gir!'s except for the presence of a kyphosis (Appendix). He enjoyed the advantages of comfortable caring parenting, however, from a happy couple and, despite his developmental problems, including operation for an oesophageal stricture and generalised 


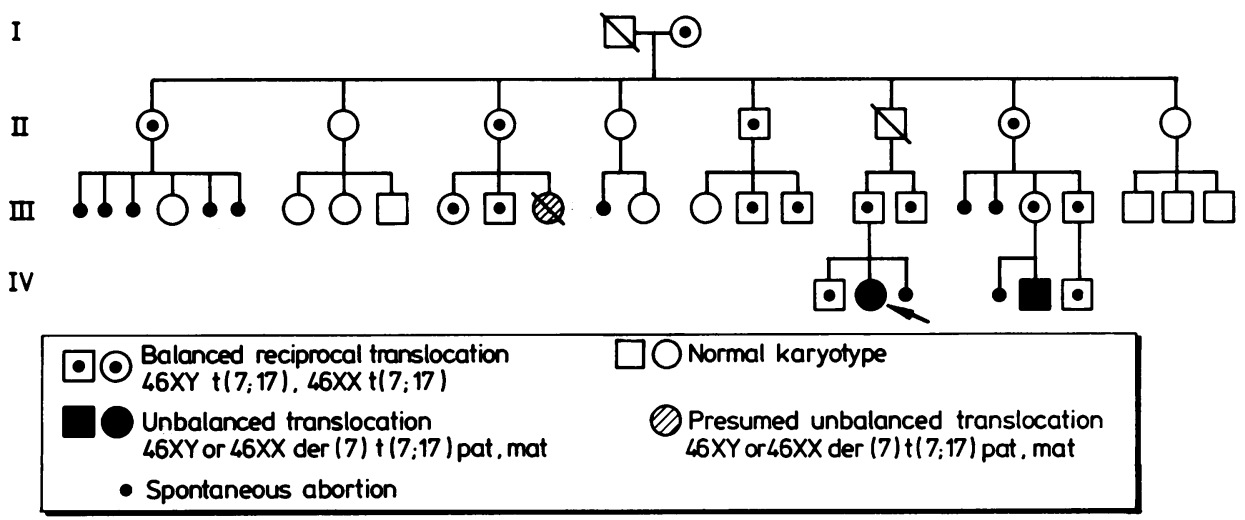

Fig. 3 The family tree of propositus.

hypotonia, was without behavioural problems. In addition to these two affected children, a further 15 members of the extended family have been identified with a balanced translocation (Fig. 3).

The clinical psychologist (ER) showed that at 43 months' old the girl had achieved a mental age equivalent to 18.8 months and an overall general quotient of 41 on the Griffiths Mental Development Scales with personal and social, hearing and speech, and eye and hand coordination scales particularly depressed. Results of a retest at 49 months were similar (mental age equivalent to 20.6 months and general quotient of 42). Her social age was equivalent to 1.94 years on the Vineland Social Maturity Scale.

\section{Treatment}

After the initial therapeutic and diagnostic interview regular close contact was made by the community psychiatric nurse for a month before a brief admission of mother and child for behavioural treatment.

The psychologist conducted all the behavioural intervention. By a multiple baseline design of four experimental conditions, which were also video recorded, the rate of the girl's eye poking behaviour was rated at intervals of one minute. The psychologist, assisted by two nurse therapists, began an intensive training session with the girl and her mother. Modelling, role playing techniques, guided participation, and praise were shown for controlling the girl's inappropriate behaviour. For example, if she rubbed or poked her eye she would be placed immediately in a corner of the room away from people, and arm splints would be put on her arms. They would be removed when her behaviour was appropriate. Positive reinforcement was offered- that is, verbal praise, hugging, and stroking of hair-for her appropriate responses-that is not poking her eye, playing, and relating more appropriately. After two and a half hours she showed that she could obey non-verbal commands such as gesture and was learning how to control her eye poking. The night staff were instructed to take four 20 minute observation recordings of her eye poking throughout the night.

Appropriate responses such as cooperative play and diminution of eye poking were concentrated on the second day. The girl's mother was instructed to take regular counts of her eye poking when leaving hospital that night. The same treatment regimen continued on days 3 and 4 when she was also rerated. The rate of her eye poking behaviour decreased to a minimum during treatment, and she showed evidence of more cooperative play behaviour.

The treatment regimen was explained to the girl's headmistress using videotaped interactions between the girl and her mother on the final day of treatment. Liaison and support were also offered to her school. The psychologist provided weekly outpatient appointments for a month to monitor treatment and focus on other aspects of the girl's inappropriate behaviour. Regular follow up for 17 months showed no evidence of eye poking behaviour and she continued to progress well at home and school.

After identification of the girl's chromosomal abnormality and when the results of the parents were available, they were seen for joint consultations between a child psychiatrist $(\mathrm{JH})$ and the clinical geneticist to explain the findings to the family, to emphasise the need for investigation of the extended family, and for treatment for the 
family. Results of this study will be reported separately.

\section{Discussion}

A newly described chromosomal aberration was seen in a girl who presented with self destructive behaviour. Medical persistence was required to complete the diagnosis, which will have profound implications for other members of her family. The behavioural treatment was useful in diminishing the self mutilation and could have been successful in a non-medical setting. Failure to make a complete medical and psychiatric appraisal of the child and her family, however, would have had serious consequences.

A broadly psychotherapeutic approach allowed the family to divulge its secrets and thus enabled the genetic diagnosis of the family in which 15 carriers of the translocation have been identified. Psychological test measures gave a rational basis to the diagnosis of mental retardation and made a level against which developmental advances, which her mother is working towards, can be sensibly measured and hence rewarded.

Psychological techniques required an appropriately supportive environment and trained nursing support for their best effectiveness in this particular predicament. The existence of another affected member of the family with a similar chromosomal make up, temperament, and developmental delay but entirely different behaviour and life course provided an example of the interposition of the predicament between the disease and its expression in behaviour.

A child psychiatrist who retains the validity of the medical model yet is accustomed to experiencing severe chronic diffuse disorders at first hand on his wards and dealing with exhausted, angry parents is in a unique position to investigate and treat such cases, and help his biotechnological colleagues. An alternative, all too often seen, is that professional exasperation with the persistent abnormal behaviour becomes attributed to psychological problems of the parents or else is seen as being a function of the mental retardation syndrome.

Child psychiatrists working in medical settings have the same rights and responsibilities for calling on the help of colleagues as any other consultants in the National Health Service without encumbering themselves with the paraphernalia of 'a team.' Proper and responsible referrals were made inter pares here to the patient's benefit. Brief admission to facilities in hospital was essential. The behavioural disorder could be regarded as the central issue for treatment and investigation rather than as a distressing limitation on the acceptability of the child in inpatient settings. Sadly, current policy for the mentally handicapped is interpreted to mean that facilities in hospitals of this sort are less likely to be available.

The medical diagnosis was of direct importance to carriers and potential cases; but it was also of profound psychotherapeutic value. It provided a rational explanation for what was otherwise a cruelly arcane fate and was a major step in coming to terms with it; it validated the medical consultation and inspired confidence and hope, and it enabled the mother to reconstruct the role that had been shaped for her both by her husband and by advisers. It was of almost no value as an explanation of the disorder for which the patient was actually referred. It is an important paradox in medicine that the proper exhibition of rational expertise may prove to be a most powerful psychotherapeutic manoeuvre.

\section{Appendix}

A series of abnormalities was noted in both children. Facial features were hypotelorism, ptosis, thin narrow face, minor epicanthal folds, long straight eyelashes, thin eyebrows, hair extending in front of the ears, prominent root of the nose, columella extending below alae nasae, mild mid face hypoplasia, everted lower lip, a tendency to protrude the tongue, prominent simple ears, and webbed neck. Both subjects showed general muscle hypotonia with a stiff gait and arms and legs held straight. They had short incurved fifth fingers, a primitive palmar crease, and proximally placed fifth toes.

We thank Dr Dian Donnai, consultant clinical geneticist, St Mary's Hospital, Manchester, for her help with this family and paper.

\section{References \\ 1 Taylor DC. The components of sickness: diseases, illnesses and predicaments. Lancet 1979;ii:1008-10. \\ 2 Taylor DC. The components of sickness: diseases, illnesses and predicaments. In: Apley J, Ounsted C, eds. One child. London: SIMP/Heinemann, 1982.}

Correspondence to Professor D C Taylor, Department of Psychiatry, University of Manchester, Manchester M27 1FG.

Received 1 March 1985 